\title{
CÁRCAVAS Y REGUEROS GENERADOS EN SUELOS SÓDICOS. PETRER (ALICANTE, ESPAÑA)
}

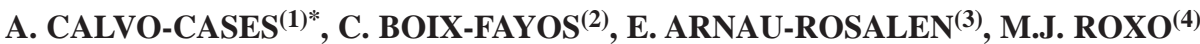 \\ ${ }^{1}$ Departamento de Geografia, Universidad de Valencia \\ ${ }^{2}$ Departamento de Conservación de Suelos y Agua (CEBAS-CSIC). Murcia \\ ${ }^{3}$ Estación Experimental de Zonas Áridas (EEZA-CSIC). Almería \\ ${ }^{4}$ Departamento de Geografia e Planeamiento Regional. \\ FCSH-Universidade Nova de Lisboa
}

\begin{abstract}
RESUMEN. Este artículo pretende mostrar las interacciones entre los procesos de piping y la formación de cárcavas y regueros en diferentes sub-sistemas (laderas y rellenos de fondo de valle) de una pequeña cuenca del SE español. Se analiza la influencia de diversas propiedades del los suelos y regolitos en el desarrollo de formas asociadas al piping. Los sistemas reguero-tunel de las laderas de badlands están principalmente relacionados con la ratio de absorción de sodio (SAR) y la conductividad eléctrica (CE) de los suelos. En contraste, en los rellenos de fondo de valle estas propiedades químicas interactúan con las discontinuidades en el gradiente hidráulico introducidas por las terrazas de cultivo y la infiltración forzada que favorecen algunas prácticas agrícolas.
\end{abstract}

\section{Gullies and rills on sodic soils. Petrer (Alicante, Spain)}

ABSTRACT. This paper concerns with the interaction between piping processes and the development of gullies and rills in different sub-systems (hillslopes and valley floor fills) present in a small catchment in SE Spain. The influence of several soil and regolith properties over forms associated to piping is analyzed. Rill-pipe systems are on the badland hillslopes mainly related with the sodium absorption rate (SAR) and the electrical conductivity $(E C)$ of the soils. In contrast at valley fills these chemical properties interact with the discontinuities of the hydraulic gradient introduced by the agricultural terraces and the forced infiltration provided by some agricultural practices.

Palabras clave: piping, cárcavas, regueros, badlands.

Key words: piping, gullies, rills, badlands.

Enviado el 10 de diciembre de 2010 Aceptado el 20 de enero de 2011

*Correspondencia: Departamento de Geografia, Universitad de Valencia. Avda. Blasco Ibáñez, 28, 46010-Valencia, España. E. mail: adolfo.calvo@uv.es 


\section{Introducción}

El SE español está salpicado de numerosas áreas con desarrollo de abarrancamientos (badlands) y, por tanto con una gran capacidad erosiva y de aporte de sedimentos a los sistemas fluviales. Todas estas áreas (Calvo-Cases et al., 1991) se han desarrollado sobre materiales arcillosos, margosos o limosos preferentemente en cuencas sedimentarias del Neógeno y su génesis suele estar ligada a importantes desniveles generados por la tectónica o cambios en la red de drenaje (Mather et al., 2002), aunque en otras ocasiones han sido los sobreusos del suelo los responsables de su desarrollo.

Tradicionalmente los procesos relacionados con la escorrentía superficial concentrada han sido asignados como característicos de las zonas de abarrancamientos, pero otros procesos, fundamentalmente movimientos en masa y piping (Calvo-Cases et al., 1991), manifiestan una notable actividad en las laderas y fondos de valle de estos sistemas.

Los regueros (rills) y su ulterior evolución en cárcavas (gullies) son formas asociadas a la escorrentía superficial concentrada y características de medios susceptibles a altas tasas de erosión, con una escasa cobertura vegetal y muy bajo contenido en materia orgánica en los suelos. Así, en los terrenos agrícolas y en las laderas de los sistemas de abarrancamientos es donde estas formas de erosión suelen adquirir una mayor relevancia. No obstante, el desarrollo de regueros y cárcavas no es necesariamente en su génesis exclusivo del flujo superficial concentrado, por el contrario está en muchas ocasiones ligado al colapso de galerías subterráneas previamente desarrolladas por la acción de la escorrentía subsuperficial concentrada.

Diversos autores (Imeson and Verstraten, 1986; Bouma, 2006) explican, en este sentido, el desarrollo de regueros hasta alcanzar las cumbres de las laderas. Esto es contradictorio con la ‘franja de no erosión' que Horton (1945) describió como característica de la partes altas de las laderas de los abarrancamientos y como necesaria para concentrar suficiente escorrentía.

El desarrollo de túneles también ha sido utilizado para explicar los procesos muy acelerados de desarrollo de cárcavas en zonas aterrazadas para el cultivo (Romero Díaz et al., 2007), del incremento de las tasas de erosión en reforestaciones realizadas utilizando terrazas (Linares et al., 2002) y en general como factor de aceleración de la erosión en suelos salinos (García Ruiz, 2010)

Aunque existen numerosos mecanismos que conducen a la formación de túneles por piping (Jones, 2010) en los medios semejantes a nuestra zona de estudio, en el SE español, son determinadas propiedades químicas de los suelos relacionadas con el contenido en sales (López Bermúdez y Torcal Sáinz, 1986; Faulkner et al., 2000; Piccarreta et al., 2006) y la modificación de la topografía introducida por determinados procesos o factores (Faulkner et al., 2008) los principales responsables de esta génesis de cárcavas y regueros dependientes del desarrollo de túneles.

La influencia de las propiedades de los materiales sobre las características de los procesos en las áreas de badlands fue inicialmente planteada por Schumm (1956) y se 
ha prestado especial atención al papel de los minerales de arcilla expandible en la generación de regueros (Bryan, 1987), así mismo, numerosos trabajos han destacado el papel de los macroporos en la formación de los regueros (Bouma y Imeson, 2000; Bouma, 2006) y de los túneles (López Bermúdez y Torcal Sáinz, 1986).

El presente trabajo pretende contribuir al estudio de la génesis de regueros y cárcavas en zonas de abarrancamientos y terrazas de cultivo a partir del estudio de las propiedades de los suelos y otros factores relacionados con el desarrollo de piping, tanto en laderas de badlands como en rellenos de fondo de valle aterrazados. El trabajo se enmarca una cuenca experimental próxima a Petrer (Alacant) en la que desde 1986 se han desarrollado diferentes estudios entorno a la dinámica de las laderas de badlands y sus posibilidades de estabilización.

\section{Zona de estudio}

Situada en el municipio de Petrer (Alacant) con $370 \mathrm{~mm}$ de precipitación media anual y $12^{\circ} \mathrm{C}$ de temperatura media, la cuenca del Barranco del Pi (Fig. 1) afluye a la

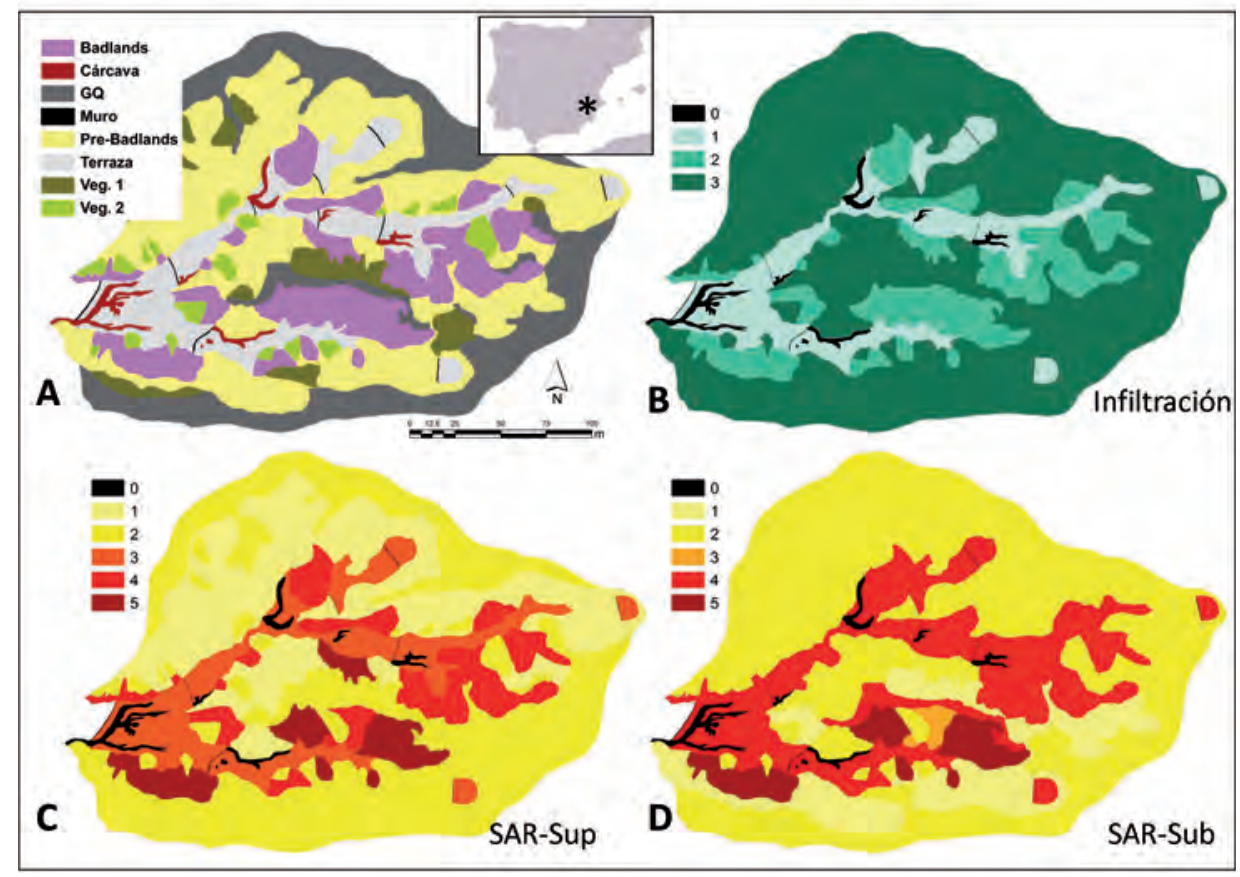

Figura 1. Situación y principales características de la cuenca del Barranco del Pi. (A) Mapa de unidades morfológicas (ver leyenda en el texto). (B) Tasas de infiltración medias en los suelos de las unidades morfológicas $\left(1=<15,2=<40\right.$ y $\left.3=<45 \mathrm{~mm} \mathrm{~h}^{-1}\right)$. (C) y (D) Distribución espacial de los valores de SAR en los horizontes superficiales y profundos del suelo, respectivamente $\left(1=<0,2,2=<0,8,3=<1,5,4=<4\right.$ y $\left.5=<7 \mathrm{meq} 100 \mathrm{~g}^{-1}\right)$. 
Rambla dels Molins, en la zona en la que esta disecta un afloramiento de materiales plásticos del Triásico superior, que junto con otras series estratigráficas del Cretácico (Senoniense) (IGME, 1978a y b), también plásticas, han alcanzado la superficie por extrusión. Así, cauce principal y afluentes han disectado varias decenas de metros de estos materiales extrusivos, apareciendo fuertemente encajados respecto de niveles de terrazas y glacis del Pleistoceno, unos $80 \mathrm{~m}$ de relieve local.

En la cuenca del Barranco del Pi afloran diversas facies de margas del Senoniense pero la disección aún no ha alcanzado los niveles Triásicos subyacentes, de modo que no ha ajustado su perfil al nivel de base local y el potencial erosivo es importante.

Como muestra la figura 1a, la cuenca está constituida por dos tributarios cuyos fondos de valle están ocupados por antiguas terrazas de cultivo, hoy abandonadas. Las prácticas agrícolas pasadas han producido un progresivo relleno de los valles y afectado a las laderas, por zapa basal, favoreciendo los procesos de erosión acelerada (Rodriguez Aizpeolea, 1992). Las laderas muestran diferentes grados de cubierta vegetal desde las totalmente desnudas (Badlands) hasta otras cubiertas por herbáceas y arbustos perennes (Figs. 2 y 3 ).

En la zona de estudio se han desarrollado diversos trabajos y una densa red de muestreo de los suelos y de medidas de infiltración con simulación de lluvia ha sido establecida para caracterizar las propiedades físico-químicas de los suelos de cada uno

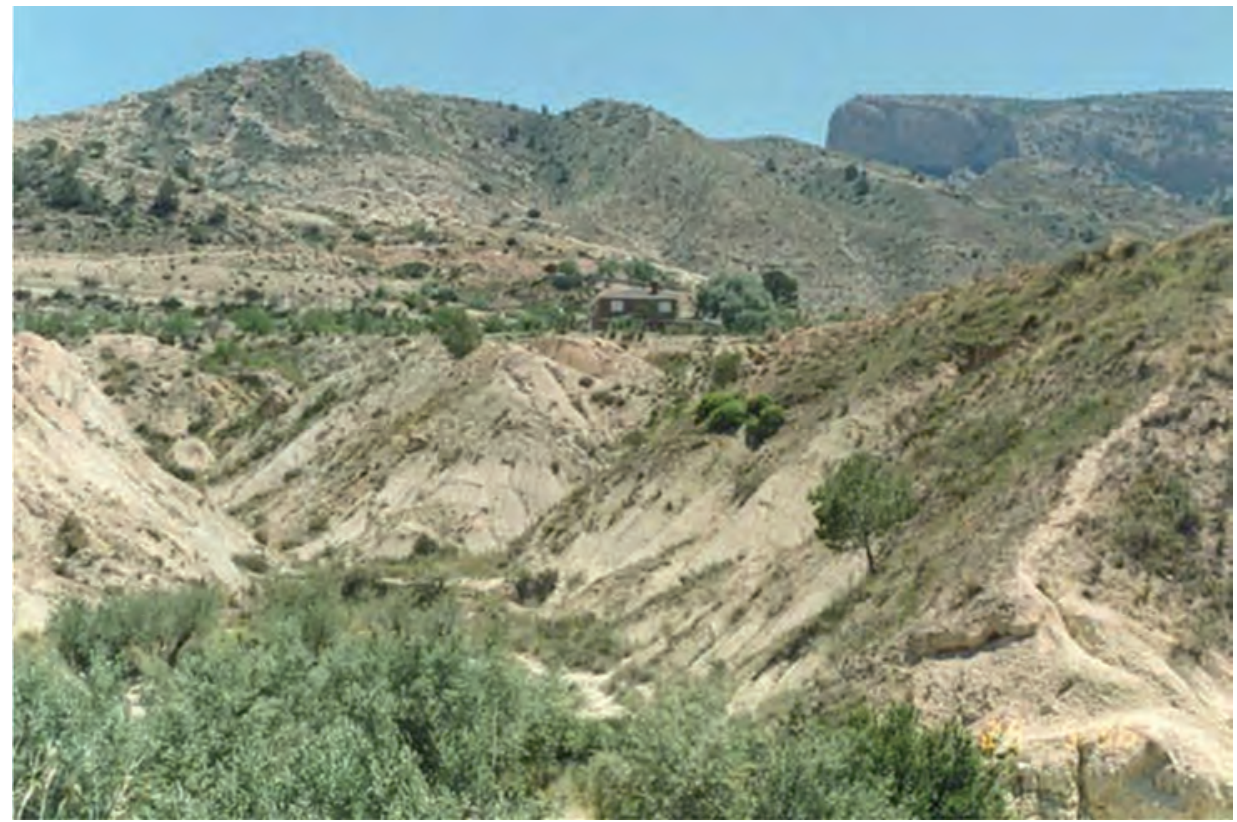

Figura 2. Vista general de la parte sur de la cuenca del Barranco del Pi. 


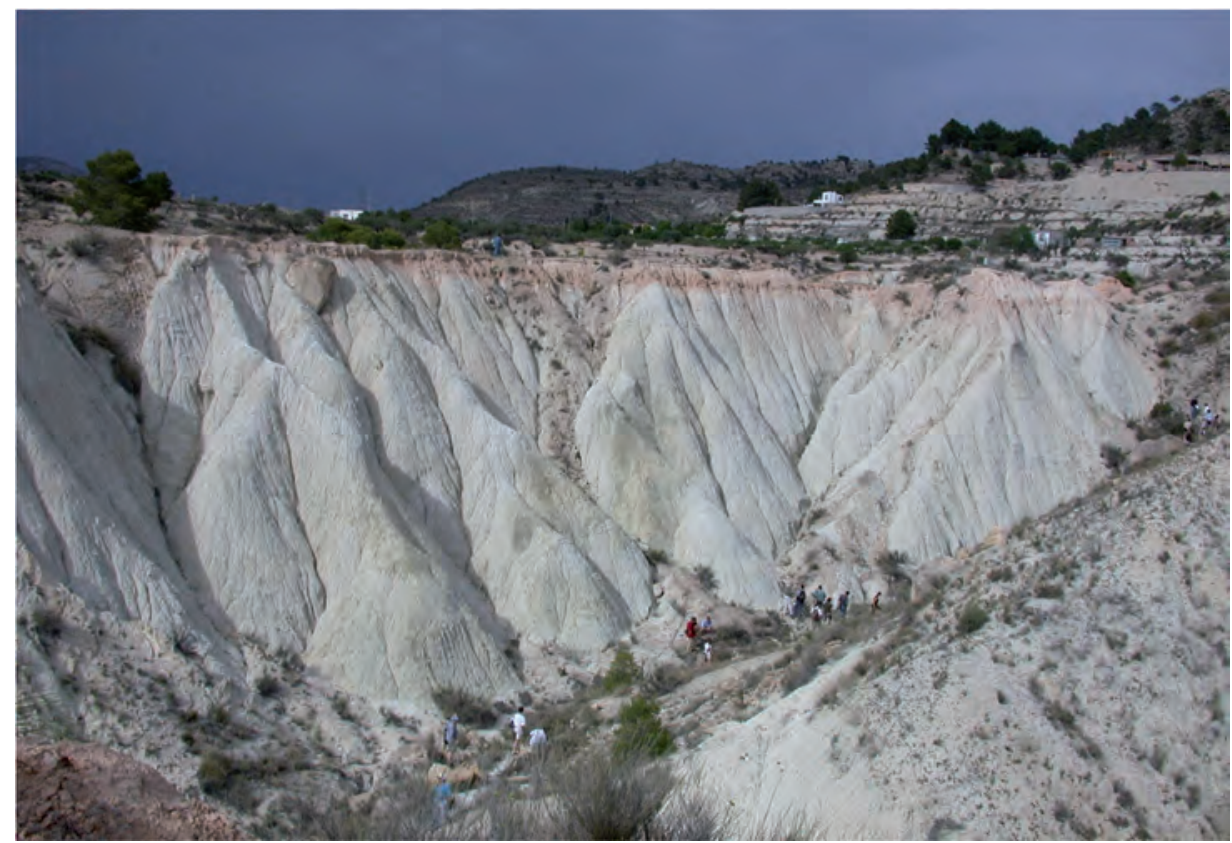

Figura 3. Laderas de badlands desarrolladas en solana en la subcuenca meridional.

de los diferentes subambientes: (i) laderas con morfología de badlands (Bd), (ii) laderas parcialmente vegetadas en proceso de degradación (PB), (iii) laderas bien cubiertas de vegetación que son restos de antiguas laderas estables (V1 y V2), (iv) cumbres en las que permanecen remanentes de depósitos de glacis cuaternarios (GQ) y (v) fondos de valle rellenos de materiales retenidos en terrazas de cultivo (Terrazas).

En las laderas de badlands se han seguido durante algunos años los cambios morfológicos para determinar la actividad de los procesos (Harvey y Calvo-Cases, 1991; Calvo-Cases et al., 1991) en una serie de parcelas seleccionadas con diferente exposición y propiedades de los materiales. Los procesos identificados incluyen la formación de grietas por expansión-contracción, coladas de barro y otros movimientos en masa superficiales, formación de regueros y formación de túneles someros (piping). Estos procesos interactúan a corto plazo y el patrón de evolución está definido por las propiedades de los materiales (Calvo-Cases et al., 1991) (Figs. 4 y 5).

En los rellenos aterrazados de los fondos de valle son relevantes en sellado superficial, la formación de pediments en la base de las laderas de badlands y la formación de cárcavas profundas a partir de las roturas de pendiente de los muros de los aterrazamientos en la que son visibles numerosas evidencia de piping en forma de túneles y puentes (Figs. 6 y 7). 


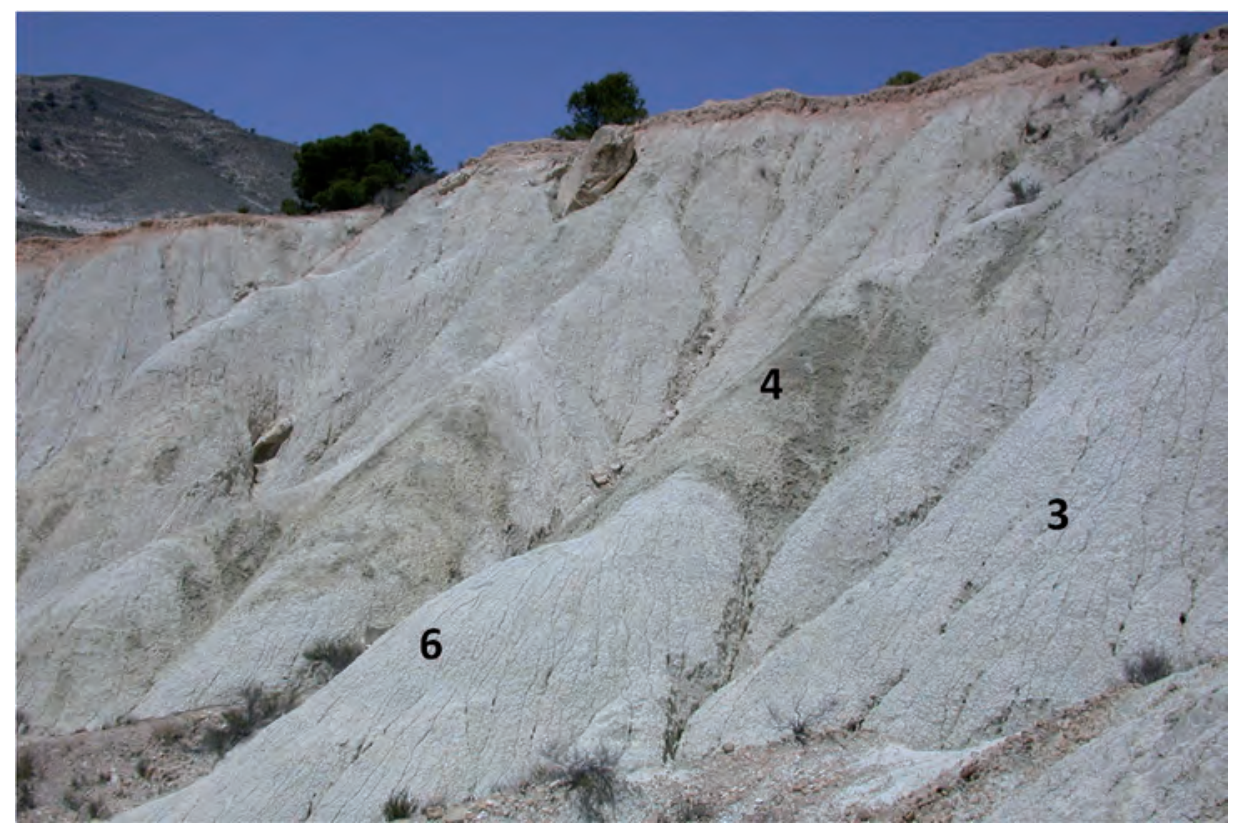

Figura 4. Detalle de las laderas de la figura 3. Los números se refieren a diferentes parcelas en las que dominan los movimientos en masa (4) o los regueros-túneles, visibles como puntos más oscuros ( 3 y 6 ).

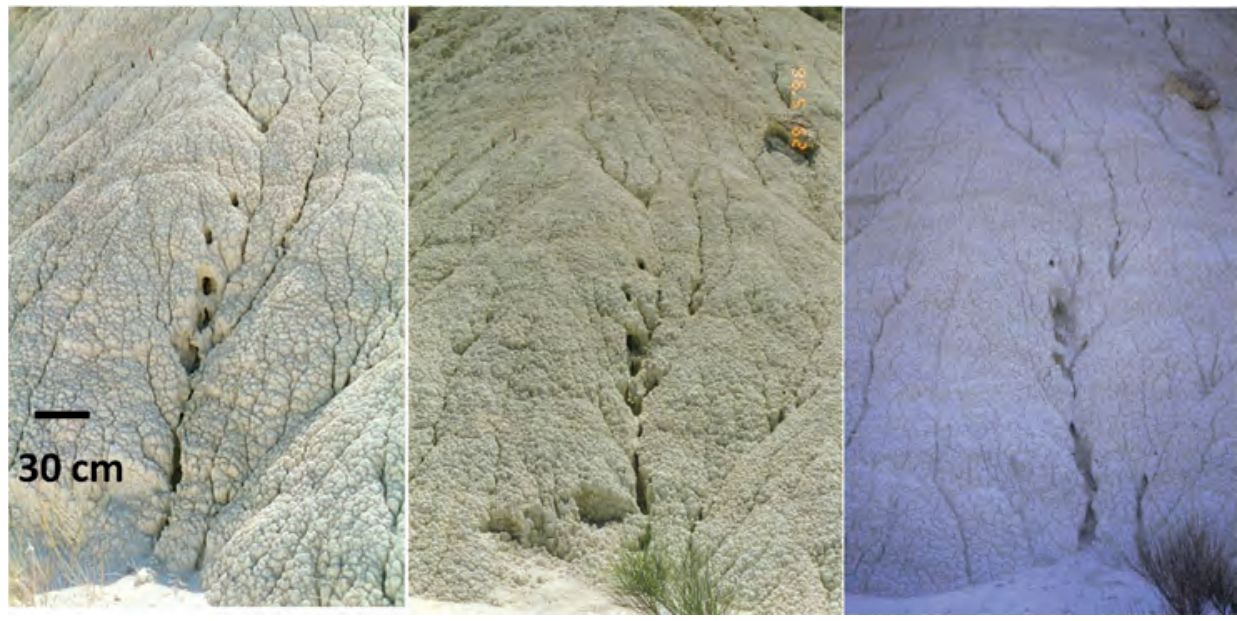

Figura 5. Secuencias de cambios en los regueros-túneles de una ladera de badlands en la umbría de la cuenca meridional. 


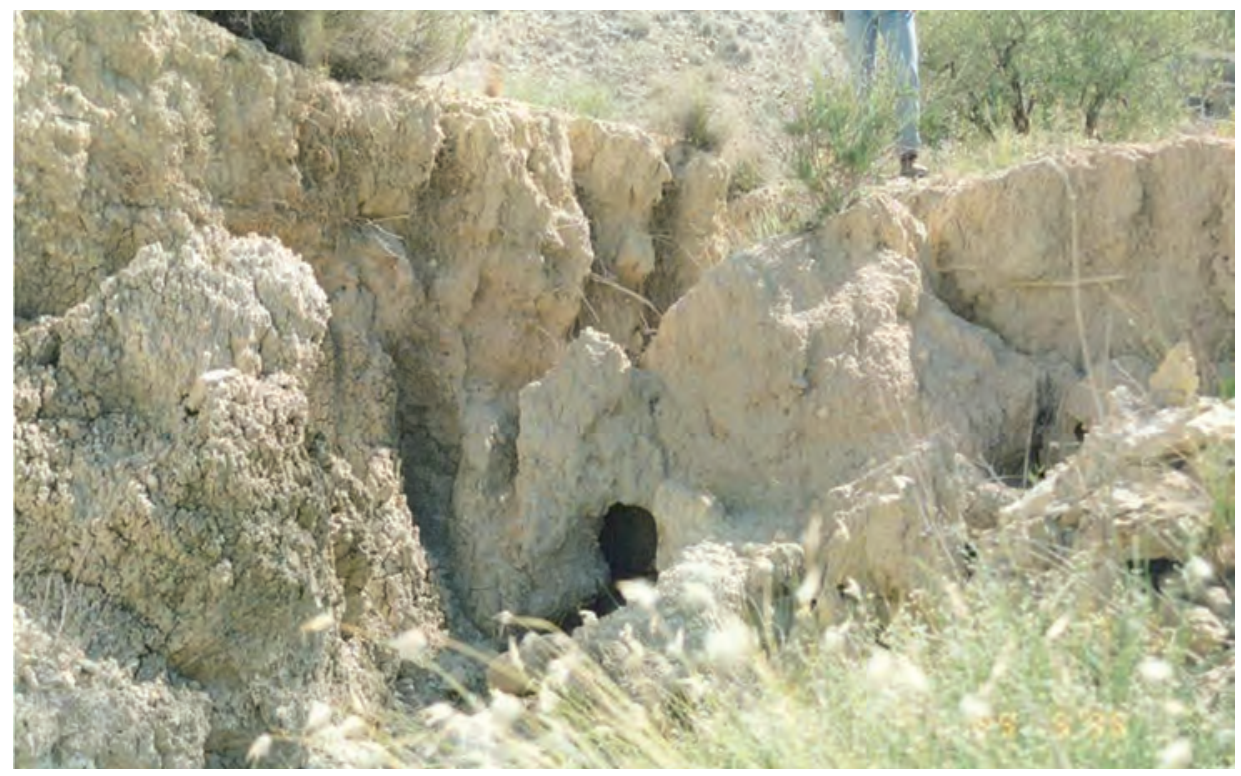

Figura 6. Túneles visibles en una de las cabeceras de cárcava de las terrazas de la subcuenca septentrional. La cárcava es aún incipiente.

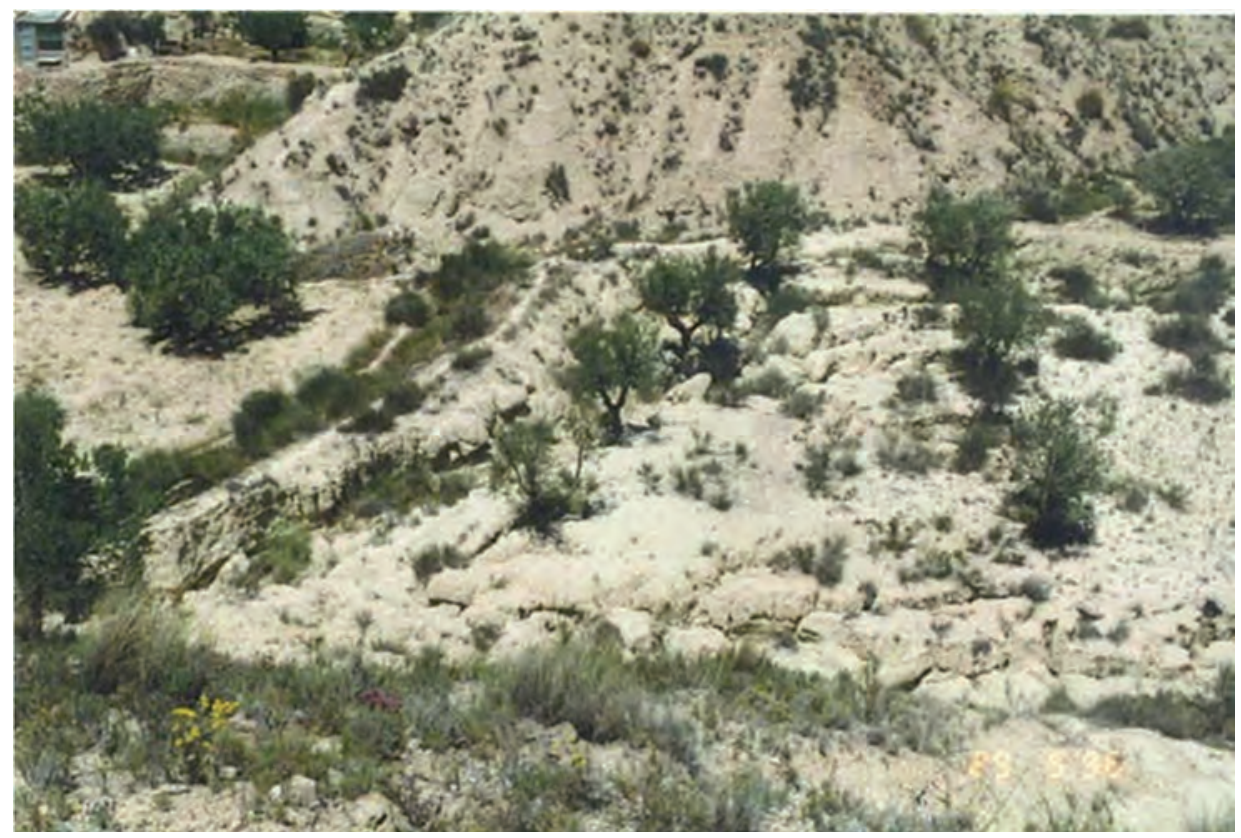

Figura 7. Terraza de cultivo abandonada en 1977 con un importante desarrollo de cárcavas. Ver figura 11. 


\section{Resultados y discusión}

\subsection{Propiedades de los suelos y regolitos}

La figura $1 \mathrm{~b}$ representa la distribución espacial de las capacidades de infiltración de los suelos en tres rangos $\left(<15,<40 \mathrm{y}<45 \mathrm{~mm} \mathrm{~h}^{-1}\right)$ obtenida a partir de treinta experimentos con simulador de lluvia según las características de Calvo et al. (1988). La menor capacidad de infiltración corresponde con los rellenos de fondo de valle, donde el sellado superficial de un suelo muy desagregado reduce la infiltración a pesar de las muy bajas pendientes $\left(<5^{\circ}\right)$. La segunda clase incluye las laderas de badlands y muestra un amplio rango de valores relacionado con las propiedades de los materiales y la exposición. El resto de ambientes mantiene tasas siempre superiores a $40 \mathrm{~mm} \mathrm{~h}^{-1}$, ligado a la cobertura vegetal y las mejores características de los suelos en estructura y contenido en materia orgánica.

En relación con otras propiedades físicas, la capacidad de retención de agua de los materiales de las laderas de badlands muestra una notable característica en un alto contenido en humedad a $\mathrm{pF} 4,2$ (marchitez permanente); $10,5 \%$ en promedio, con valores entre 6,4 y $14,0 \%$.

La mineralogía de las arcillas, ha sido obtenida para diecisiete muestras de regolito en otras tantas laderas de badlands en la cuenca sur. Los minerales el grupo de la smectita (montmorillonita) abundan en seis de las muestras analizadas (más de 10\%) y están presentes en la mayoría (Fig. 8) en proporciones altas.

Las propiedades químicas de los suelos muestran diferencias entre las unidades de la cuenca. A partir de 35 puntos de muestreo distribuidos por las laderas y fondos de valle de la cuenca sur, se han determinado $\mathrm{Ca}++, \mathrm{Mg}++, \mathrm{K}+, \mathrm{Na}+, \mathrm{pH}$ y $\mathrm{CE}$ (Conductividad Eléctrica, en $\mathrm{mS} \mathrm{cm}^{-1}$ ), ente otros. Tal como muestra la figura 8 el sodio es el catión dominante en la mayoría de las muestras analizadas.
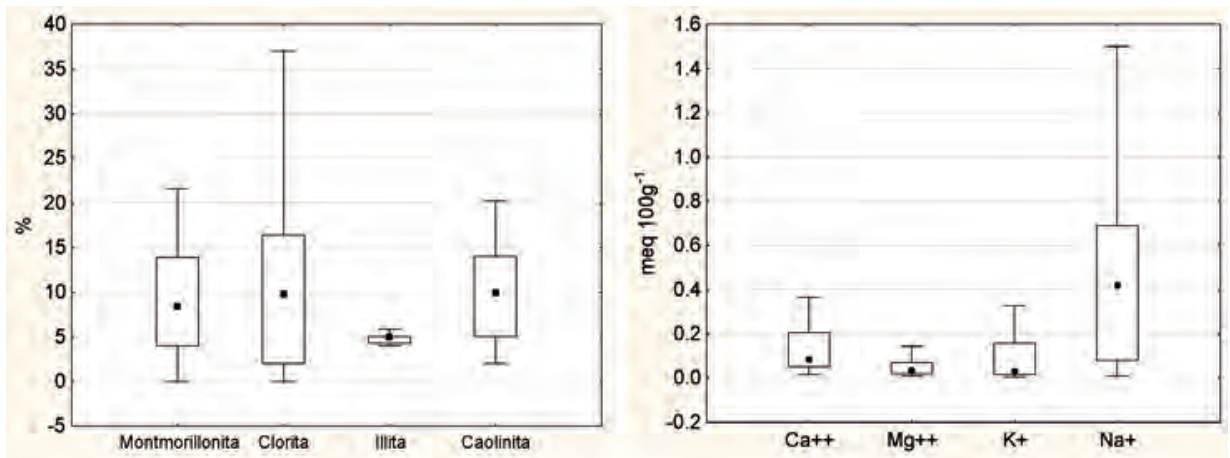

Figura 8. Distribución de los valores relativos de la mineralogía de la arcillas y los cationes en los suelos. Los rectángulos cubren del 25 al 75\% de los datos y los puntos centrales corresponden a la mediana. 

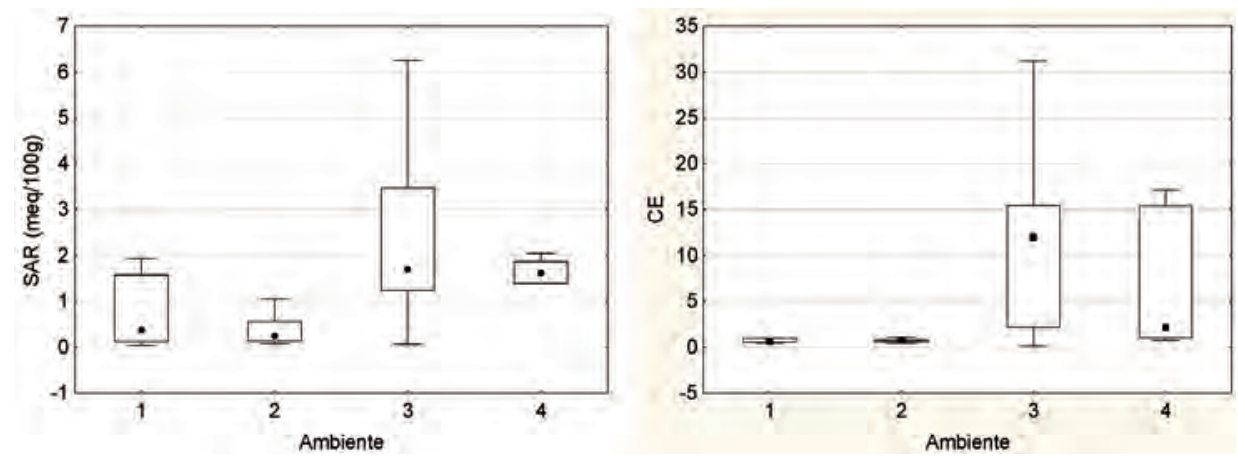

Figura 9. Distribución de la SAR y CE en diferentes ambientes de la cuenca ( $1=$ Cumbres sobre depósitos cuaternarios, $2=$ Prebadlands, $3=$ Badlands, $4=$ Fondos de valle $)$.

La distribución espacial de la ratio de absorción de sodio (SAR) en las diferentes unidades o sub-ambientes de la cuenca y correspondiente a los horizontes superficiales de los suelos (Fig. 1c) y a los horizontes C o R (Fig. 1d), destaca el predominio de suelos sódicos en las laderas de badlands y en los fondos de valle. Por el contrario, los suelos más desarrollados y protegidos por la vegetación muestran los valores más bajos.

Tanto la SAR como la CE marcan diferencias claras entre los cuatro sub-ambientes de los que proceden las muestras analizadas (Fig. 9), de modo que podemos establecer

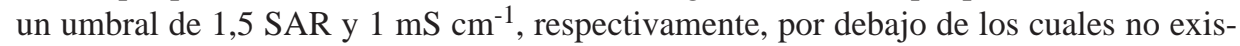
ten ambientes proclives a la formación de regueros o de cárcavas. Por encima de estos valores encontramos tanto las laderas de badlands como los rellenos de fondo de valle.

\subsection{Procesos en las laderas}

En la cuenca de estudio las laderas suponen el $86 \%$ de la superficie total, y las laderas sin ninguna cobertura vegetal, con morfología de badlands, alcanzan al 16\%. Estas laderas están caracterizadas por una considerable actividad erosiva. A partir de simulaciones de lluvia en parcelas pequeñas (c.a. $0,24 \mathrm{~m}^{2}$ ) la concentración de sedimentos en la escorrentía supera en el $90 \%$ de los casos los $20 \mathrm{~g} \mathrm{l}^{-1}$ y en tasas de erosión el $75 \%$ de los experimentos supera los $500 \mathrm{~g} \mathrm{~m}^{-2} \mathrm{~h}^{-1}$, con una tasa de erosión media de $1798 \mathrm{~g} \mathrm{~m}^{-2}$ $\mathrm{h}^{-1}$. Estas tasas de erosión son considerablemente menores en los restantes ambientes: 2,7 en las laderas con vegetación, 60 en los pre-badlands y 162 en los pedimentos de los fondos de valle. Proporcionalmente al área representada por cada unidad y partiendo de los datos de simulación de lluvia, las laderas de badlands aportan el $89 \%$ de la producción de sedimentos en la cuenca generados por salpicadura y arroyada difusa.

Medidas entre 1988 y 1997, a partir de varillas de erosión en 6 parcelas de badlands, las tasas medias de rebajamiento de suelo han sido de entre 2 y $7 \mathrm{~mm}^{2} \tilde{n}^{-1}$. 
Las tasas obtenidas con simulación de lluvia son consecuencia de la actividad de la salpicadura, la arroyada difusa y, en el caso de las varillas de erosión, está también representada la arroyada concentrada en regueros, aunque posiblemente infravalorada por las características propias del muestreo.

No obstante en la cuenca han sido identificados otros procesos (Harvey y CalvoCases, 1991) cuya actividad y tasas son de evaluación difícil con los métodos aplicados. Así, en las laderas labradas sobre margas con mayor contenido en smectita estos procesos se combinan con la actividad de movimientos en masa superficiales (Calvo-Cases y Harvey, 1996). Además, en las laderas en materiales con mayor contenido en sodio son características formas asociadas al piping (Figs. 4 y 5) Normalmente se trata de galerías de algunos centímetros de diámetro que discurren paralelas a la superficie, siguen el patrón de grietas y suelen derivar en regueros (Fig. 5); son semejantes a los drenajes descritos por Gerits et al. (1987). En algunos casos y en los márgenes de regueros grandes cuyo encajamiento produce un escalón en la base de la ladera, se han observado galerías verticales de dimensiones semejantes (Fig. 4, número 3). La figura 10 muestra una relación positiva entre un índice de actividad del piping obtenido para 6 laderas de badlands a partir de la interpretación de fotografías secuenciales (Calvo-Cases y Harvey, 1996) y la relación de absorción de sodio (SAR). Así mismo, esa relación es inversa con el contenido en montmorillonita. Tal como han mostrado otros autores (Faulkner et al., 2000) es el contenido en sales dispersantes el principal factor que posibilita el desarrollo de pipes en estas laderas de badlands.

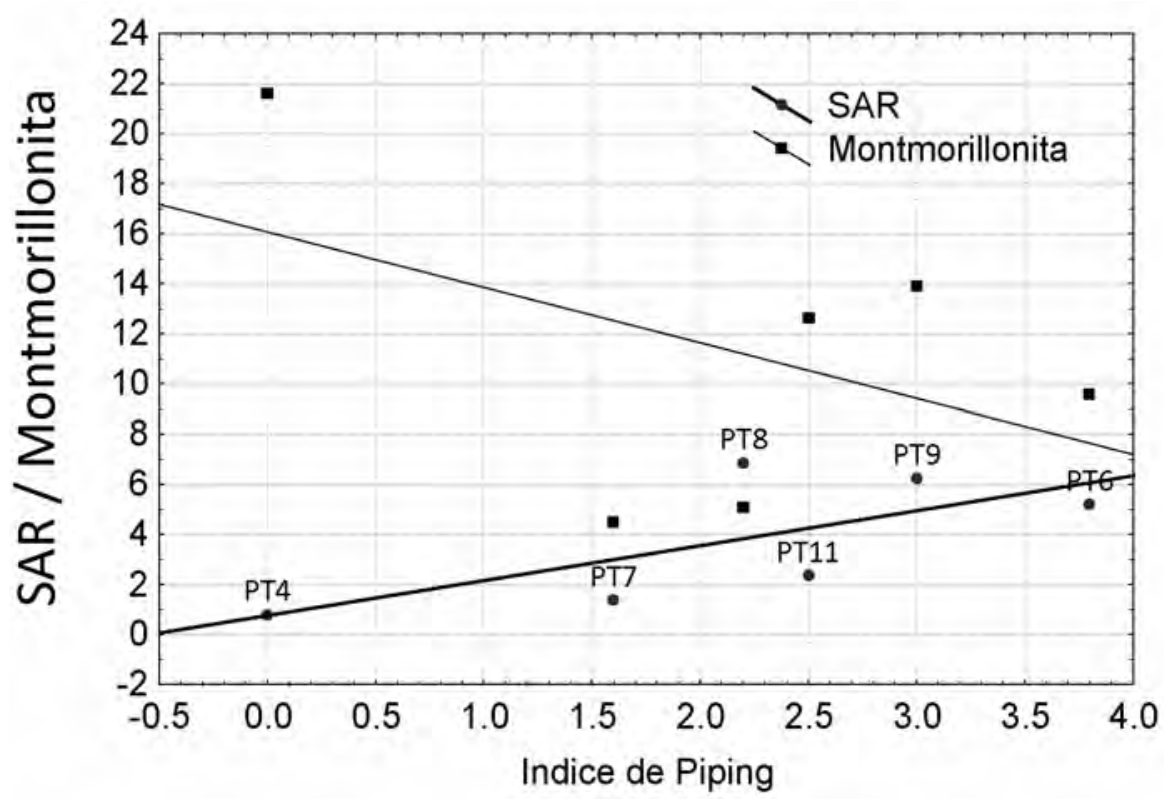

Figura 10. Relaciones entre el desarrollo de túneles por piping y la SAR y proporción de montmorillonita. 


\subsection{Procesos y formas en los fondos de valle}

Los fondos de valle de la cuenca corresponden a una serie de antiguas terrazas de cultivo abandonadas desde 1977 en el caso de las que aún se conservan como tales y unos 30 años antes en el valle de la cuenca sur, de las que apenas quedan vestigios (Rodriguez Aizpeolea, 1992).

La técnica de cultivo en la zona incluye el mantenimiento por encima del muro de la terrazas de un caballón que discurre paralelo a este y actúa como presa de parte del agua de escorrentía forzando el riego, al tiempo que favorece la sedimentación. La agricultura tradicional incluía sistemas para el drenaje de los excesos de escorrentía (Asins Velis, 2009) de los que apenas quedan vestigios en la cuenca.

En la situación actual la mayoría de las terrazas están disectadas por cárcavas que parten de la rotura de los muros (Figs. 1a, 6 y 7) y alcanzan el fondo original con profundidades de hasta $2 \mathrm{~m}$. En las cabeceras de algunas de las cárcavas es visible (Fig. 6) su prolongación en túneles de unos 50 a $70 \mathrm{~cm}$ de diámetro y algunas de las cárcavas son discontinuas en superficie mostrándose como una sucesión de colapsamientos interconectados por túneles.

En la terraza más baja de la figura 1a, es donde las cárcavas han adquirido un mayor desarrollo durante los años de estudio. Así, para la elaboración de la figura 11 se reconstruyó

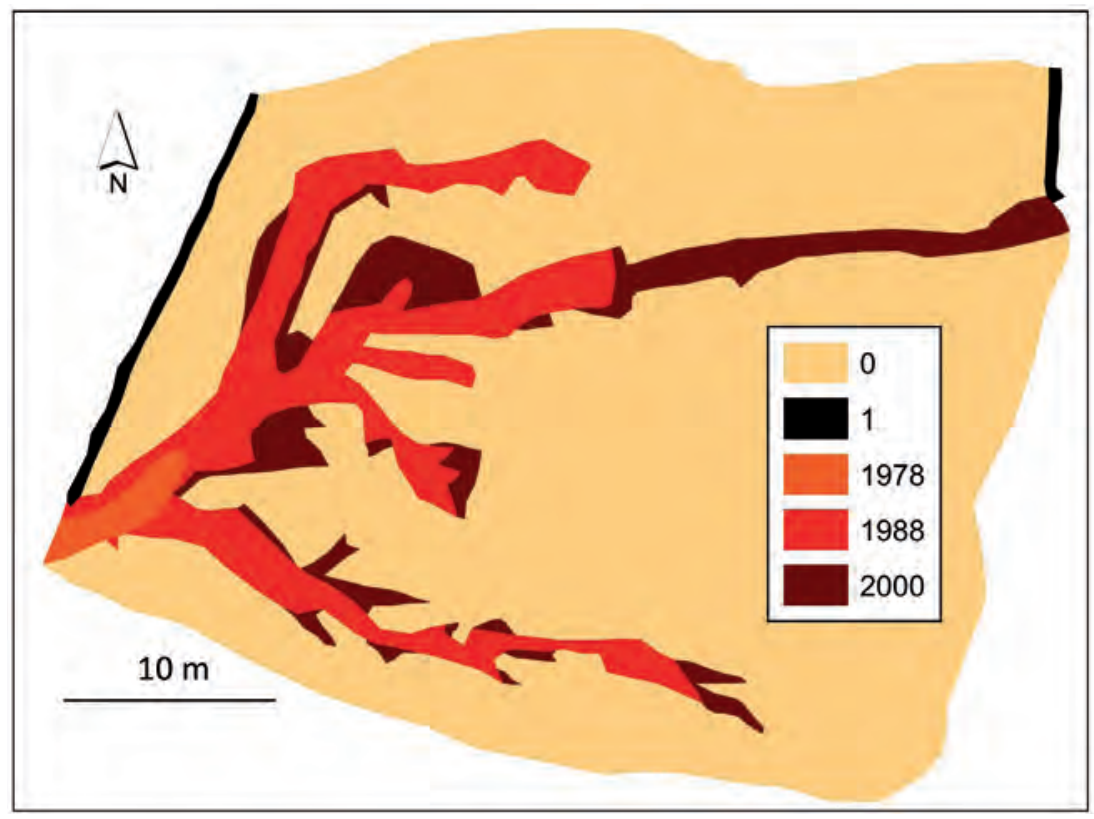

Figura 11. Evolución de las cárcavas desarrolladas en la terraza de la parte baja de la cuenca (ver figura 7) tras el abandono de la agricultura en $1977(0=$ superficie del bancal, $1=$ muros). 
el área de las cárcavas a partir de fotografías aéreas tomadas en 1978. En 1988 se realizó un levantamiento topográfico detallado para la medición de los volúmenes de las cárcavas y este se repitió en 2000.

El volumen erosionado entre 1978 y 1988 fue de 394,6 $\mathrm{m}^{3}$, mientras que el volumen entre 1978 y 2000 fue de 440,3 m³ , equivalentes a 374 y $190 \mathrm{t} \mathrm{ha}^{-1}$ año ${ }^{-1}$, respectivamente y en relación al área que ocupa la terraza $\left(1266,7 \mathrm{~m}^{2}\right)$. Los resultados de la volumetría de las cárcavas muestran que el desarrollo más rápido corresponde a la primera década, mientras en los 12 años posteriores el incremento fue de apenas $45,7 \mathrm{~m}^{3}$ (Fig. 11) correspondientes al ensanchamiento de algunas cárcavas por colapsos laterales y a la prolongación del ramal principal (norte). Pero este ramal es un canal muy somero con profundidad de solo algunos centímetros, mientras que las ya desarrolladas en la primera década alcanzan hasta $1 \mathrm{~m}$ de profundidad.

En consecuencia, tras el abandono del cultivo en 1977, en un año se formó una cárcava de unos $7 \mathrm{~m}$ de longitud, observable en la fotografía aérea de 1978 (Fig. 11). En solo 10 años más, la red de cárcavas alcanzo su desarrollo con casi 400 $\mathrm{m}^{3}$ de suelo evacuados, para posteriormente ralentizar su velocidad erosiva. En la interpretación de estos datos es necesario acudir al piping como principal proceso del desarrollo de las cárcavas, bien visible en el campo, como se muestra en las figuras 6 y 7 .

Tal como se ha cartografiado en la figura 1a, basada en fotografías aéreas estereoscópicas de 1991 a escala 1:3000, prácticamente todas las terrazas de cultivo abandonadas en los años 70 están disectadas por cárcavas que parten de un lateral del muro y en algunos de los casos visiblemente desarrolladas en paralelo a dicho muro. En todas estas terrazas se han observado formas asociadas al piping con túneles o puentes o colapsos circulares que denuncian la existencia de una red de galerías que a media que va colapsando se transforma en cárcavas.

Las evidencias de formación de túneles y su posterior evolución en cárcavas están asociadas a la existencia de elementos dispersables en los materiales que constituyen el relleno de las terrazas. Procedentes de la erosión de las laderas de badlands, estos rellenos muestran valores de SAR y CE (Figs. 1c, 1d y 9) superiores a los de los suelos con cubierta vegetal y, especialmente en la CE similares a los del material original que constituye el regolito de las laderas sin vegetación. Al efecto de las propiedades del material hay que añadir el de la rotura del gradiente hidráulico del suelo que implica el escalonamiento en terrazas. En unas condiciones en que el caballón construido en el borde de las mismas obliga a una infiltración abundante forzada por el espesor de la lámina de agua. Aunque las tasas de infiltración medidas en la actualidad (tras el abandono de la agricultura) son muy bajas (Fig. 1b) podemos suponer que serian mayores cuando los campos estuviesen labrados.

En los campos en producción de los alrededores, sobre materiales similares, los colapsamientos del suelo próximo a los muros de las terrazas son frecuentes (Asins Velis, 2009) tras eventos de lluvia que incrementan el peso de la bóvedas de los túneles. 
En los campos abandonados estos colapsamientos no son subsanados con el relleno de los huecos, de modo que se inicia un desarrollo progresivo y acelerado de cárcavas, inicialmente siguiendo el patrón de los túneles preexistentes, y paralelos a los muros, y posteriormente en la dirección del gradiente hidráulico marcado por las cabeceras y laterales verticales de las cárcavas. A media que la superficie de los antiguos campos va sellándose por la desagregación del suelo y la sedimentación de arcillas y limos, se reduce la capacidad de infiltración y es posible el drenaje superficial, por la falta de obstáculos y la mejora introducida por la red de cárcavas. El piping pierde relevancia y el desarrollo pasa a ser responsabilidad del retroceso de las cabeceras por escorrentía superficial durante eventos de magnitud alta.

\section{Conclusiones}

La zona estudiada contiene muchas de las características de algunos valles frecuentes en el SE español en los que el piping es un proceso muy relevante en el desarrollo de cárcavas y regueros, tanto en los fondos de los valles como en las laderas de badlands.

El desarrollo de túneles poco profundos y paralelo a la superficie en las laderas de badlands es necesario para explicar la formación de muchos regueros y especialmente de los que discurren por las divisorias y, por tanto, son independientes del área de drenaje para su formación. La condición necesaria es la existencia de materiales dispersables normalmente por su elevado contenido en sodio, alta SAR o alta CE. La relación inversa con el contenido en arcillas expandibles sugiere que la acción de estas puede dificultar la formación o la permanencia de los túneles.

En los fondos aterrazados son la rotura del gradiente hidráulico, la infiltración forzada y, también, las propiedades del material, los principales factores para explicar el desarrollo acelerado de cárcavas tras el abandono de la agricultura. Con el paso del tiempo desde el abandono, en el caso concreto de nuestra zona de estudio, el piping deja de ser relevante con la impermeabilización de los antiguos campos y es sustituido por arroyada superficial en el desarrollo de las cárcavas.

\section{Agradecimientos}

Queremos manifestar nuestro agradecimiento a Mario Payà y su familia, por la siempre desinteresada e inestimable ayuda y facilitación del trabajo de campo. Mucho del trabajo aquí incluido ha sido realizado con el soporte económico de los proyectos NAT89-1072-C06-04 y AMB93-0844-C06-04 del Plan Nacional de I+D (CICYT). 


\section{Referencias bibliográficas}

ASINS VELIS, S., (2009). El paisaje agrario aterrazado: Dialogo entre el hombre y el medio en Petrer (Alicante). PUV, 359 p., Valencia.

Bouma, N. A., (2006). Rill Initiation and Development in Relation to Dynamic Soil Properties. Tesis doctoral. Universidad de Amsterdam. 317 p., Amsterdam.

BoumA, N.A. Y IMESON, A.C., (2000). Investigation of relationships between measured field indicators and erosion processes on badland surfaces at Petrer, Spain. Catena, 40: 147-171.

BRYAN, R.B., (1987). Processes and significance of rill development. En: Rill Erosion: process and significance (Bryan R.D., ed.), Catena Suppl., 8: 1-15.

Calvo, A., Gisbert, B., Palau, E., Romero, M., (1988). Un simulador de lluvia portátil de fácil construcción. En: Métodos y técnicas para la medición de procesos geomorfológicos (Sala, M. y Gallart, F., eds.), S.E.G. Monografía 1: 6-15.

Calvo-Cases, A., Harvey, A.M., Paya-Serrano, J., (1991). Process interactions and badland development in SE Spain. En: Soil erosion studies in Spain (Sala, M., Rubio, J.L. y García-Ruiz, J.M., eds.), Geoforma Ediciones, 75-90 p., Logroño.

Calvo-Cases, A., Harvey A.M., (1996), Morphology and development of selected badlands in Southeast Spain: implications of climatic change. Earth Surface Processes and Landforms, 21: 725-735.

FAulKner, H., SPIVEy, D., AleXANDER, R., (2000). The role of some site geochemical processes in the development and stabilisation of three badland sites in Almería, Southern Spain. Geomorphology, 35: 87-99.

Faulkner, H., Alexander, R., Zukowskyj, P., (2008). Slope-channel coupling between pipes, gullies and tributary channels in the Mocatán catchment badlands, Southeast Spain. Earth Surf. Process. Landforms, 33: 1242-1260.

GARCiA Ruiz, J.M., (2010). The effects of land uses on soil erosion in Spain: A review. Catena, 81: 1-11.

Gerits, J.J.P., Imeson, A. C., Vestraten, J.M., BryAn, R.B. (1978). Rill development and badland regolith properties. En: Rill erosion: process and significance (Bryan, R.D., ed.), Catena Suppl. 8: 141-160.

Harvey, A. M., Calvo-Cases, A., (1991). Process interactions and rill development on badland and gully slopes. Z. Geomorph. Suppl.-Bd., 83: 175-194.

HorTOn, R.E., (1945). Erosional development of streams and their drainage basins; hydrophysical approach to quantitative morphology. Bulletin of the Geological Society of America, 56: 275-370. 
IGME (1978a). Mapa Geológico de España (E: 1/50,000). Elda (Hoja 871). Instituto Geológico y Minero de España, Madrid.

IGME (1978b). Mapa Geológico de España (E: 1/50,000). Alicante (Hoja 872). Instituto Geológico y Minero de España, Madrid.

IMESON, A.C., VERTSRATEN, J.M., (1986). Erosion and sediment generation in semi-arid and Mediterranean environments: the response of soils to wetting by rainfall. Journal of Water Resources, 5: 388-418.

Jones, J. A. A., (2010). Soil piping and catchment response. Hydrol. Process., 24: 15481566.

Linares, R., Rosell, J., Pallí. L., RoQue, C., (2002). Afforestation by slope terracing accelerates erosion. A case study in the Barranco de Barcedana (Conca de Tremp, NE Spain). Environmental Geology, 42: 11-18.

LóPez Bermúdez, F., TORCAL SÁINZ, L., (1986). Procesos de erosión en túnel (piping) en cuencas sedimentarias de Murcia (España). Estudio preliminar mediante difracción de rayos X y microscopio electrónico de barrido. Papeles de Geografía Física, 11: 7-20.

Mather, A., Stoker, M., Griffiths, J. S., (2002). Quaternary landscape evolution: A framework for understanding contemporary erosion, Southeast Spain. Land Degrad. Develop., 13: 89-109.

Piccarreta, M., Faulkner, H., Bentivenga, M., Capolongo, D., (2006). The influence of physico-chemical material properties on erosion processes in the badlands of Basilicata, Southern Italy. Geomorphology, 81: 235-251

RodrigueZ-AizPEOLEA, J., (1992). Un ejemplo de la influencia de uso y abandono de bancales de fondo de canal en la evolución de Badlands (Petrer-Alacant). Estudios de Geomorfología en España. Actas del II Congreso Nacional de Geomorfología, 211-219 p, Murcia.

Romero díaz, A., Marín SAnleandro, P., SÁnchez Soriano, A., Belmonte Serrato, F., FAULKNER, H., (2007). The causes of piping in a set of abandoned agricultural terraces in southeast Spain. Catena, 69: 282-293.

ScHumm, S. A., (1956). The role of creep and rainwash on the retreat of badland slopes. American Journal of Science, 254: 693-706. 